\title{
El artículo en las relativas introducidas por con en el siglo XVIII: contextos y evolución*
}

\author{
The article in the relative clauses headed by con throughout \\ eighteenth century: contexts and evolution
}

\author{
Francisco Javier Vellón Lahoz \\ Universitat Jaume I \\ vellon@uji.es \\ ORCID iD: https://orcid.org/0000-0002-7752-2793
}

RESUMEN: A partir de un corpus formado por textos cercanos al polo de la inmediatez comunicativa (sobre todo, cartas privadas) del siglo XVIII, el artículo ofrece los resultados de un análisis variacionista en torno a un cambio gramatical que empieza a extenderse en la citada centuria: la inserción del artículo en las cláusulas de relativo oblicuas, en este caso, las introducidas por la preposición con. Los datos muestran que, pese a que la forma etimológica, sin artículo, continúa siendo dominante, algunos contextos lingüísticos comienzan a favorecer la difusión de la variante innovadora, seleccionados como significativos por el análisis de regresión.

Palabras clave: sociolingüística histórica, variación sintáctica, cláusulas relativas, español, textos de inmediatez comunicativa, lingüística de corpus, siglo XVIII.

ABSTRACT: Based on a corpus of ego-documents (mainly private letters) from the eighteenth century, the article offers the results of a variationist analysis about a grammatical change that starts in this century: the insertion of the article in relative clauses with preposition, specifically in clauses headed by preposition con. The data show that, despite etymological form, without article, is still the dominant variant, several linguistic contexts begin to favor the diffusion of the innovative variant, selected as significant by the regression analysis.

\footnotetext{
* El presente trabajo se enmarca dentro del proyecto de investigación "Dimensiones estructurales, sociales e idiolectales del cambio lingüístico: nuevas aportaciones desde la sociolingüística histórica al estudio del español", financiado por el Ministerio de Economía, Industria y Competitividad (Ref. FFI2017-86194-P) y la Universitat Jaume I (Ref. UJI-B2017-01), cuyos detalles pueden encontrarse en la siguiente dirección electrónica: $<\mathrm{http}$ ://sociolinguisticawe.wixsite.com/sociolinguisticauji $>$.
} 
Keywords: historical sociolinguistics, syntactic variation, relative clauses, Spanish, immediacy texts, corpus linguistics, eighteenth century.

\section{INTRODUCCIÓN}

El presente artículo versa sobre un fenómeno de variación centrado en la presencia del artículo en las proposiciones de relativo oblicuas, concretamente en las introducidas por la preposición con. Se enmarca, por tanto, en la diacronía de la categoría artículo, en la ampliación de sus funciones originales y en la paulatina consolidación de sus contextos de uso. Así, frente a la especialización de los demostrativos — origen etimológico de la nueva categoría romance- en la función de deíxis exofórica, el artículo encuentra su ubicación en el sistema en la identificación unívoca del referente (Leonetti, 1999: 800), aspecto que se revela como un factor decisivo en su nuevo uso en las relativas oblicuas, como se verá en las páginas siguientes.

La elección del siglo XVIII para ubicar el inicio del proceso de innovación no es arbitraria. Como señala Sánchez Lancis (2012), la periodización de la lengua debería atender más a los cambios gramaticales que a los factores externos. Desde esta convicción, el autor plantea que el siglo XVIII es una frontera entre el español clásico y el moderno, puesto que comienzan algunos de los fenómenos que se generalizarán durante las centurias posteriores. Nuestros trabajos relacionados con otros fenómenos de cambio gramatical confirman esta condición de la centuria ilustrada (Blas Arroyo, Porcar y Vellón, 2013: 36; Blas Arroyo y Vellón, 2015: 93)

Sirva como ejemplo, para el caso que nos ocupa, la relación epistolar de Pedro Rodríguez Campomanes, durante los últimos años del mencionado siglo, con diversos personajes de la época. En ella se observa el incipiente cambio que se estaba produciendo en el sistema de las cláusulas de relativo oblicuas, en este caso, en las introducidas por la preposición con:

(1) [...] con el seguro de nuestra pronta voluntad, con la que quedo su más fiel amigo y servidor (Francisco de Rivera a Rodríguez Campomanes, Campomanes. Epistolario, 1776).

(2) Conozco la buena voluntad con que Vm. ha llevado delante del trono el memorial de mi yerno (Rodríguez Campomanes a Pedro de Lerena, Campomanes. Epistolario, 1789) $)^{1}$.

En efecto, mientras que en (1) aparece la solución innovadora, con la presencia del artículo, el ejemplo (2) ofrece el testimonio de la variante tradicio-

\footnotetext{
${ }^{1}$ En los ejemplos, se resalta en cursiva el relativo y la preposición, así como, en cada caso, el factor analizado.
} 
nal, sin el determinante, aún mayoritaria, pero que comienza a ver amenazada su preponderancia por la estructura que, con el tiempo, se convertirá en la forma no marcada de la oposición. A este respecto, no deja de ser sugerente que en la correspondencia posterior de Jovellanos, en los últimos decenios del siglo y comienzos del siguiente, se incremente la presencia del relativo con artículo:

(3) Cuídate y vive seguro de mi inalterable cariño, con el que soy siempre tu más fino hermano Gaspar (Jovellanos a su hermana, Jovellanos. Correspondencia, 1778).

(4) [...] aprovecho tan oportuna ocasión para ofrecerme a sus órdenes con la más fina voluntad, con la que ruego a nuestro Señor [...] (Jovellanos a Cienfuegos, Jovellanos. Correspondencia, 1800).

El presente artículo tiene como objetivo analizar este fenómeno de variación que se da en el ámbito de las relativas oblicuas ${ }^{2}$ y que Brucart (1999: 496) considera "uno de los fenómenos más complejos de la gramática española [...] sometido a un considerable margen de variación diatópica e incluso idiolectal". Si bien en este caso el interés se centrará en las cláusulas de este tipo regidas por la preposición con, en otros trabajos anteriores se han observado ya los factores que condicionan la inserción del artículo en las oblicuas introducidas por la preposición en (Blas Arroyo y Vellón, 2017 y 2018; Vellón y Moya, 2017), las más numerosas en nuestro corpus ( $\mathrm{y}$ también en los utilizados por las diversas investigaciones sobre el tema, que serán comentadas posteriormente — vid. infra § 2-).

La finalidad del trabajo será no solo confirmar los datos obtenidos en las aproximaciones precedentes, sino revisar, y acaso ampliar, algunas de las tendencias que se adivinaban en los albores del cambio, y que ahora, con otra de las formas implicadas, las segundas en número de ocurrencias, pueden arrojar resultados más concluyentes. En nuestro corpus, las ocurrencias con artículo introducidas por en eran 127, lo que representaba el $17 \%$ del conjunto de relativas oblicuas encabezadas por tal preposición (754). En el caso de las que serán objeto de estudio en este artículo, el número total es de 672, de las cuales 101 son con artículo (15\%). Como se verá más tarde $(\S 3)$, para el presente trabajo se han incorporado más ocurrencias de textos, con similares características discursivas a los que componen nuestro corpus, procedentes de otros corpus, tal como se detallará en el mencionado apartado.

${ }^{2}$ El objetivo del trabajo son las cláusulas de relativo oblicuas con antecedente externo ("es el barco con que vinisteis/es el barco con el que vinisteis"), en las que el artículo es una marca de concordancia con el antecedente. Son las únicas estructuras de este tipo en las que puede existir variación. Quedan fuera, por tanto, las relativas sin antecedente expreso ("estuve con el que me dijiste"), en las que el artículo es el antecedente del pronombre y en las que no es posible la alternancia, ni, por ello, el análisis variacionista. 
Todos los estudios diacrónicos sobre el fenómeno (Brucart, 1999; Girón Alconchel, 2006; Guzmán Riverón, 2012) señalan que en el siglo XVIII se hace evidente la extensión de esta estructura. De hecho, las muestras de épocas anteriores son escasas y poco representativas (Keniston, 1937: $\S \S 15.158$ y 15.228; Eberenz, 2000: 368; Lapesa, 2000: 398). En un corpus del siglo XVII similar al manejado en estas páginas, las cifras obtenidas de dicha centuria apuntan en esa misma dirección, pues solo hemos encontrado cinco ejemplos de la variante con artículo en las oblicuas con en, y tres en las introducidas por con. A pesar de ello, las muestras de este último caso ya indican algunas de las vías de penetración de la forma innovadora, concretamente la distancia del antecedente y la presencia del artículo (5) (vid. infra § 5), como revela el siguiente ejemplo:

(5) [...]cuánto contento me sea ver los afectuosos y encendidos afectos del corazón de vuestra merced, con los que este tiene Nuestro Señor tan enriquecido (Cartas de Luisa Acosta).

La evolución del proceso a partir del siglo XVIII va a dar lugar a un paulatino arrinconamiento de las formas etimológicas, sin artículo, y a la consecuente extensión de la variante innovadora, lo que acabará de consolidarse en el siglo $\mathrm{XX}^{3}$.

Antes de ofrecer los principales resultados e implicaciones teóricas que de ellos se derivan en el apartado 5, en la sección siguiente se presenta un breve estado de la cuestión sobre la variable lingüística. A este seguirá una descripción más detallada de los textos que componen el corpus (§ 3 ), así como de la metodología utilizada $(\S 4)$.

\section{El artículo en las Relativas Oblicuas. APORTACIONES SOBRE LA DiA- CRONÍA DEL FENÓMENO}

La aparición del relativo con artículo tiene su origen, según señala Lapesa (2000: 388 y ss.), en la construcción formada por el pronombre anafórico latino IS + relativo, y la posterior conversión a la categoría de pronombre demostrati-

\footnotetext{
${ }^{3}$ En nuestra investigación (Vellón y Moya, 2017) sobre las relativas oblicuas introducidas por las preposiciones monosilábicas ( $a$, con, de, en, por), a partir de diez periódicos de difusión nacional, los datos fueron concluyentes: de las 14.572 ocurrencias obtenidas de relativas oblicuas, tan solo 1066 (el 7,21\% del total) mantuvieron la forma original sin artículo. De ellas, 867 estaban introducidas por en $(81,33 \%), 142$ por $\operatorname{con}(13,32 \%), 38$ por $d e(3,54 \%), 19$ por a $(1,78 \%)$ y ningún caso con la preposición por. Por su parte, el estudio de Santana Marrero (2004) a partir del Macrocorpus de la norma lingüística culta de las principales ciudades del mundo hispánico, con notable presencia del español de América, arroja resultados diferentes a los expuestos, con una pervivencia de la forma sin artículo muy alta: el $75,89 \%$ frente al $24,10 \%$ en el caso de las oblicuas con en, y datos más igualados en el de con, pues la forma con artículo aparece en un $53,59 \%$. Estas cifras demuestran que la variación puede estar sometida a un notable factor dialectal.
} 
vo ILLE QUI. La transformación del paradigma de los demostrativos, que da origen a la categoría artículo en el latín vulgar, plantea una primera cuestión referida a las relativas sin antecedente externo: ¿en qué categoría se incluye y cuál es la función del elemento que antecede al relativo?

Puesto que dicha estructura no es el objeto de este trabajo, baste con recordar que para algunos autores prevalece la postura de la gramática de la RAE (en su versión de 1931, como recuerdan Gili Gaya, 1970: 304, § 231; y Girón Alconchel, 2006: 1495), según la cual, en la mencionada distribución, el artículo mantiene el valor pronominal etimológico (Bosque y Moreno, 1990: 44; Briz y Prunyonosa, 1987: 104-105). Frente a ello, la mayoría de los estudiosos considera que se produce un proceso de gramaticalización por el que "el pronombre originario se ha convertido en un nuevo artículo" (Hernández, 1986: 479), es decir, "un proceso de fosilización" (Marcos Marín, 1980: 237) de la construcción artículo + relativo que confluye en la aparición de un relativo compuesto el que (similar al caso de el cual, vid. Elvira, 1985: 308-309), en el que "el artículo obligatorio se ha ido propagando en el español moderno a la forma que" (Lope Blanch, 1998: 575).

El siguiente aspecto, este sí más relevante para los objetivos de la presente investigación, es determinar si existen diferencias entre las construcciones de relativas oblicuas con y sin artículo. Para Alarcos (1994: 108, § 146) se trata de construcciones sinónimas ya que "el artículo no añade ningún valor significativo". En términos parecidos se manifiestan otros autores, que explican la alternancia como un fenómeno sintáctico que no afecta a la estructura del enunciado (cf. García García, 1990: 56; Trujillo, 1990: 27-28).

A pesar de ello, en la bibliografía sobre el tema numerosos trabajos se detienen en determinar qué factores son relevantes para la alternancia entre construcciones, tanto para explicar qué distribuciones contribuyeron a la extensión de las formas con artículo, como para discernir en qué contextos del español actual se mantiene la forma del relativo simple. Entre estos últimos cabe destacar el trabajo de Brucart (1999: 494-495), quien considera que la naturaleza definida del antecedente favorece el mantenimiento de la variante sin artículo, aspecto en el que coincide Santana Marrero (2004) y que matiza Girón Alconchel (2006: 1499), pues considera que es sencillo encontrar ejemplos de lo contrario: "Llega un momento en la vida en que nada tiene importancia". El resto de autores que se han ocupado del tema han puesto de relieve la incidencia de otros factores para explicar la variación, como la caracterización semántica del antecedente (- humano/+ humano) (Porto Dapena, 1997: 24-25), el tipo de relativa (López García, 1994: I, 440-442), la función del pronombre relativo (Martínez, 1989: 154), el grado de determinación de todo el SN antecedente (Balbachán, 2011), razones estilísticas (Rivero, 1991: 73-75) y otros condicionantes relacionados con la gramática cognitiva (Delbecque, 2011). 
Más interés, si cabe, tienen para nuestro trabajo las aportaciones centradas en el análisis diacrónico del fenómeno, sobre todo las que se ocupan del siglo XVIII, centuria en la que, como se dijo (vid. $\S 1$ ), comienza la expansión de la construcción de relativo con artículo. Tanto en el caso de Guzmán Riverón (2012: 189-191), con un corpus procedente de textos americanos del $C O R D E^{4}$, como en el de Girón Alconchel (2006: 1528 y ss.), con textos cercanos a la norma culta, se enfrentan al problema de la escasez de ocurrencias de cláusulas oblicuas con artículo: 20 introducidas por la preposición con ha consignado Guzmán Riverón, lo que representa apenas un $5 \%$ del total (381 ejemplos), mientras que en el caso de Girón Alconchel son 32 (un $7 \%$ del total). Con tan escasa muestra, es lógica la afirmación en torno a la imposibilidad de formular generalizaciones en lo que afecta a los contextos que favorecen la forma innovadora, por lo que "hemos de contentarnos con ofrecer ejemplos de ritualización de su uso en determinadas formulaciones, en este caso propias de textos de corte administrativo-judicial" (Guzmán Riverón, 2012: 190).

Tanto la aportación de Girón Alconchel como la de Guzmán Riverón se fundamentan en dos aspectos que contrastan con la que contiene el presente artículo (vid. infra $\S \S 3$ y 4). Por una parte, estos corpus se limitan a muestras discursivas de índole preferentemente formal, lo que condiciona la obtención de ocurrencias en un fenómeno que parece ajustarse al modelo de "cambio desde abajo" ("sistemático, que aparece primero en el lenguaje corriente propio y representa la acción de factores internos, lingüísticos", Labov, 1996: 145), tal como indicamos en nuestros trabajos precedentes (Blas Arroyo y Vellón, 2017 y 2018). Por otra, la metodología, de raíz descriptivista, está basada en el recuento de frecuencias y porcentajes de una única variante, sin tener en cuenta el principio laboviano de responsabilidad ante los datos (accountability) (Labov, 1972), según el cual, para evaluar la variación es necesario dar cuenta no solo de una forma, sino también de aquellas otras que compiten con la anterior en la disputa por un mismo campo refererencial o funcional. Además, la consideración aislada de los factores condicionantes puede determinar su significación real, lo que impide observar las potenciales relaciones entre sí para la explicación de la variabilidad.

\section{CORPUS}

El presente trabajo forma parte de un proyecto de sociolingüsitica histórica (vid. supra nota al título) para que el que se ha elaborado un corpus de textos,

\footnotetext{
${ }^{4}$ Por la relación que realiza la autora, la mayoría de las referencias procede de textos cultos, especialmente relaciones, documentos jurídico-administrativos, géneros historiográficos, lo que puede haber condicionado la obtención de ocurrencias.
} 
que van desde el siglo XVI al siglo XX, pertenecientes a un registro cercano al polo de la inmediatez comunicativa (Oesterreicher, 2004). Para este artículo la muestra incluye las ocurrencias procedentes del siglo XVIII, la mayoría del género epistolar y pertenecientes a españoles de diversa procedencia social, cultural y dialectal, que han escrito sus cartas tanto desde España como desde América, y que comprenden desde asuntos familiares hasta otros de índole pública, por lo que en las misivas se pueden distinguir diversos grados de intimidad/solidaridad entre los interlocutores. El corpus de la mencionada centuria también compila, aunque en menor número, muestras de otros géneros de características similares, como diarios, memorias, testimonios en procedimientos judiciales, etc.

Frente a las consideraciones en torno a los corpus realizadas en el apartado anterior, el que constituye la referencia de este trabajo se ajusta al modelo más cercano a la caracterización oral, por lo que los trabajos de sociolingüística histórica lo han considerado como el más adecuado para el estudio de la variación y del cambio lingüístico (Poplack y Malvar, 2007; Poplack y Dion, 2009; Elspass, 2012).

El resultado es un corpus formado por 1.242 .588 palabras y con miles de textos escritos por 1345 escritores diferentes. A partir de él se han obtenido 672 ocurrencias, de las cuales 101 corresponden a la variante con artículo $(15 \%)$ y 571 a la forma etimológica ( $85 \%)$.

Con el fin de ampliar la muestra, y así obtener resultados más significativos, se han añadido ocurrencias procedentes de otros dos corpus habituales en este tipo de estudios: el CORDE de la RAE y el Corpus del español de Mark Davies (<http://www.corpusdelespanol.org/>). Para mantener la continuidad respecto al corpus propio, la selección se ha realizado a partir de los siguientes criterios:

a.- los textos pertenecen a géneros discursivos similares a los descritos anteriormente, cercanos al polo de inmediatez comunicativa;

b.- en cada uno de ellos se han rescatado tanto las formas innovadoras como las variantes tradicionales, lo que garantiza la coherencia del análisis variacionista.

El corpus final, sobre el que se realizado el presente estudio, ha arrojado un total de 959 ocurrencias, 134 de las cuales lo hacen con la estructura con el que $(14 \%)$.

En la mayoría de los casos, los textos han sido transcritos por filólogos, aunque también se han aceptado los editados por historiadores sociales, siempre que hayan manifestado haber respetado la literalidad de los textos, con la única salvedad de la modernización de la ortografía y la puntuación para facilitar la lectura. Esta consideración, que los invalidaría para trabajos centrados en cuestiones fónicas o de grafías, no afecta a estudios, como el presente, cuyo objetivo son las estructuras gramaticales (Bergs, 2012). 


\section{Metodología}

La primera fase de la investigación se dirigió a la aplicación del programa de concordancias Wordsmith 6.0 al corpus descrito en el apartado anterior para seleccionar las ocurrencias de la variable a partir de los criterios que se indican más abajo ${ }^{5}$.

Posteriormente, con el fin de determinar los contextos que favorecen la variante innovadora, objetivo de este artículo, se procedió a la codificación de los diferentes factores lingüísticos y extralingüísticos. A continuación se ofrece la relación de factores, cuya explicación y ejemplificación más detallada se reserva para aquellos que hayan resultado significativos en un apartado posterior (infra $\S 5)$. Conviene precisar que la elección de estos factores responde tanto a las aportaciones de la bibliografía sobre el tema (supra $\S 2$ ), como a nuestros trabajos previos en estructuras gramaticales similares. Precisamente, a partir de esta experiencia se han incorporado todos los condicionantes que aparecieron como significativos para la expansión del relativo con artículo en las cláusulas oblicuas de relativo, así como los que, a merced de los datos descriptivos obtenidos en su momento, mostraban una tendencia que podría resultar determinante en este sentido. Por la misma razón, se han desestimado los factores lingüísticos que nunca han aparecido como explicativos del fenómeno de variación ${ }^{6}$.

En lo que se refiere a los factores lingüísticos, los hay que afectan al antecedente, y en este trabajo son: lema; distancia (adyacente/no adyacente); grado de determinación (determinado/indefinido/no determinado); género (masculino/femenino); número (singular/plural); categoría semántica (abstracto/concreto); paralelismo estructural — priming effect 1 - (presencia del artículo/ausencia); paralelismo estructural — priming effect 2- (presencia de la preposición con en el antecedente/ausencia).

En cuanto a los factores vinculados con la cláusula relativa, son los siguientes: sentido de la relativa (modo/otros); sintaxis del sujeto (explícito/omitido); función del relativo (CC/suplemento); modalidad oracional (afirmativa/negativa); tiempo y modo del verbo — presente/pasado/futuro/condicional/subjuntivo/formas no personales - (recodificados, tras la comprobación de frecuencias observadas en la tabla de contingencia, como presente/futuro/pasado-otros, para facilitar el análisis de regresión); tipo de relativa (explicativa/especificativa).

\footnotetext{
${ }^{5}$ Lógicamente, para el caso de los corpus señalados - CORDE y Davies-, la selección de variantes se realizó a partir de los mecanismos ofrecidos por la aplicación de búsqueda de cada una de las páginas web.

${ }^{6}$ Entre ellos merecen destacarse los siguientes: función y categoría del antecedente, modalidad de la oración principal, grado de impersonalidad de la cláusula de relativo, correferencialidad de los sujetos (principal/relativa), sintaxis oracional, es decir, la aparición de la relativa en la oración principal, en la subordinada o en la coordinada.
} 
Finalmente, se codificó un factor extralingüístico, el tiempo, conceptualizado a partir de la década ( 0 a 9 ) en que se escribieron los textos, que en todos nuestros trabajos se ha mostrado como el más significativo de esta categoría y, sin duda, el que mejor revela el proceso temporal del cambio lingüístico. También se incluyó el factor antecedente, puesto que es relevante para la siguiente fase del análisis.

La matriz de datos obtenida tras la codificación, tanto de las ocurrencias con artículo como de las innovadoras, sin artículo, fue sometida a un análisis de regresión logística de efectos mixtos, mediante el programa Rbrul (Johnson, 2009), para lo cual las ocurrencias de la variable mayoritaria - las relativas oblicuas encabezadas por con que - se opusieron a las de la forma con artículo - con el que-, considerada como la variante de referencia (application value). A la hora definir cada factor para el análisis de regresión, el eje temporal ("década") se consideró como continuo, mientras que los relacionados con el antecedente ("lema") se interpretaron como aleatorios. El resto fueron factores categóricos, es decir, mediante la oposición de opciones fijas: explicativas/especificativas, masculino/femenino, etc.

A partir de este análisis se obtienen los datos de los factores considerados como significativos en la aparición de las formas con relativo + artículo en las cláusulas de relativo oblicuas introducidas por la preposición con. Estos datos se miden en términos probabilísticos (valores P.), que se sitúan entre dos extremos: 0 (nulo favorecimiento de una variante) y 1 (selección de la variante). Se considera que los índices por encima de 0,50 favorecen la presencia de una variante y los situados por debajo lo desfavorecen. El programa Rbrul permite analizar los casos según factores aleatorios; en este caso, el factor de este tipo, antecedente, se analiza con el resto de factores, de manera que el modelo de regresión solo considera significativo un factor si el resultado es más fuerte que el ejercido por dichos factores de agrupación.

\section{RESUltados y ANÁLISIS}

En este apartado se analizarán los resultados obtenidos tras la aplicación del programa de regresión al corpus disponible (vid. supra § 3) y que pueden verse en la Tabla 1. El análisis contemplará los factores seleccionados como significativos para la difusión de la variante marcada, en este caso la forma con artículo (con el que), tanto los lingüísticos como el relativo al eje temporal. Pero también se comentarán algunos condicionantes que, aunque no han sido señalados por Rbrul a la luz de la muestra disponible, revelan tendencias que podrían tener relevancia explicativa ante una muestra más amplia y que pueden ser sintomáticos respecto a una dirección del cambio lingüístico que se consolidará 
en épocas posteriores (investigación que está en curso en la actualidad a través del estudio de las ocurrencias obtenidas en los siglos XIX y XX).

\begin{tabular}{|l|c|c|c|c|}
\cline { 2 - 5 } \multicolumn{1}{l|}{} & $\mathrm{N}$ & $\%$ & TOTAL N & P. (ANTECEDENTE) \\
\hline DISTANCIA DEL ANTECEDENTE & & & & \\
\hline No adyacente & 48 & 45.7 & 105 & .83 \\
\hline Adyacente & 86 & 10 & 854 & .16 \\
\hline Rango & & & & 67 \\
\hline PRIMING (PRESENCIA ARTíCULO EN ANTECEDENTE) & & & & \\
\hline No presencia & 100 & 35.4 & 282 & .77 \\
\hline Presencia & 34 & 5 & 677 & .23 \\
\hline Rango & & & & 54 \\
\hline SENTIDO DE LA RELATIVA & & & & \\
\hline Instrumental-Otros & 109 & 24.7 & 380 & .75 \\
\hline Modal & 25 & 5.2 & 566 & .25 \\
\hline Rango & & & & 50 \\
\hline TIPO DE RELATIVA & & & & \\
\hline Explicativa & 121 & 43.8 & 276 & .88 \\
\hline Especificativa & 13 & 2 & 683 & .11 \\
\hline Rango & & & & 77 \\
\hline DÉCADA (VAR. CONTINUA) & & & & \\
\hline Continuous logodds & & & & +10.394 \\
\hline
\end{tabular}

TABLA 1.-Factores seleccionados como significativos por Rbrul tomando como variable dependiente la forma con + artículo + que. Con el factor antecedente como variable aleatoria: desviación, 351.101; AIC (Akaike’s Information Criterion) ${ }^{7}$, 351.253; proporción, .14.

\subsection{Factores lingüísticos}

El análisis de regresión determina que hay cuatro factores significativos (como se ha dicho, con valores probabilísticos P. por encima de .50), es decir, que favorecen la variante con artículo: el tipo de relativa (P. relativas explicativas: .88, como valor más favorecedor), la distancia del antecedente (P. no adyacencia: .83, el segundo en la línea citada), la presencia/ausencia del artículo en el antecedente ( $\mathrm{P}$. ausencia: .77) y el sentido de la relativa (P. instrumental: .75).

${ }^{7}$ El Criterio de Información de Akaike con corrección para muestras pequeñas (AICc, por sus siglas en inglés) refleja la relación entre el ajuste del modelo a sus datos así como su complejidad. Los valores más bajos revelan un equilibrio más ajustado entre las citadas dimensiones. El AICc no indica si una variable es significativa, sino que permite concluir si el modelo de referencia pierde información determinante si se suprime la variable (vid. Claes, 2014: 175). 


\subsubsection{Presencia del artículo y distancia del antecedente}

El grado de determinación del SN antecedente ha sido presentado como uno de los factores asociados a la aparición de la variante con artículo en las oblicuas de relativo. Brucart (1999: 495) plantea que los antecedentes definidos favorecen la variante simple, lo mismo que señala Girón Alconchel (2006: 1530). En nuestro trabajo sobre las oblicuas introducidas por en en el siglo XVIII (Blas Arroyo y Vellón, 2017), observamos que este contexto aparecía como significativo, aunque con un rango explicativo bajo (P. 59). Por su parte, en el dominio de las formas determinadas, las diferencias frecuenciales entre los contextos indefinidos y definidos eran muy bajas $(18 \%$ y $17 \%$, respectivamente), lo que explicaría que, en la presente investigación, el programa no lo haya seleccionado como significativo.

En este caso, para el análisis se distinguieron tres posibilidades: sin determinación del antecedente (6 y 7), con determinación definida, en la que se incluyen tanto las ocurrencias con artículo como aquellas en las que el antecedente aparece actualizado por otros determinantes como posesivos, demostrativos y otras formas identificativas $(8,9,10$ y 11), y con determinación indefinida (12 y 13$)$ :

(6) [...] pues tienes para todo entendimiento con el que se gobierna todo (Cartas desde América).

(7) [...] será tanto mi esmero en servirla como es imbiolable cariño con que amo a Vm. (Historias de América. La inmigración en tinta y papel).

(8) [...] y en el interín recibe mi corazón con el que pido a Dios te guarde (Die Private Korrespondenz).

(9) [...] tengo ya mis medianos créditos con que poder mantenerme (Cartas desde América).

(10) [...] me dieron una prueba de aquel favor con que V.E. se sirvió honrarme (Campomanes, Epistolario).

(11) vuestra merced me dice que se halla en el convento de San Basilio con un religioso [...], cuya noticia, con la que vuestra merced me participa su desamparo (Cartas desde América).

(12) Remito otra remeza con la que Vmds. se satisfagan (Al recibo de esta).

(13) [...] será Dios servido de darme alguna cortedad con que pueda siquiera ponerme a tu vista (El hilo que nos une).

El análisis multivariante con las tres posibilidades mostró que este factor no es significativo para la estructura innovadora. Se obtuvo el mismo resultado tras la recodificación del factor en dos valores: "definidos"/"otros". Sin embargo, los datos estadísticos ofrecían algunos indicios llamativos, y así en la categoría "otros" el $25.4 \%$ de las ocurrencias corresponden a antecedentes de la variante con el que, frente a las de la categoría "definido", que solo representan el $11 \%$. Puesto que el factor "presencia/ausencia del artículo" sí que aparece 
como significativo, y dado que es un factor vinculado con el de la "determinación" - todos los antecedentes con artículo aparecen concretamente en la categoría de "definidos", lo que refleja una coalescencia entre formas y explicaría por qué el programa no la ha seleccionado como significativa-, es el más indicado para explicar las cifras comentadas.

La presencia del artículo en el antecedente (lo que afecta a todo el SN y no solo al núcleo, vid. Ojea, 1992: 33; Brucart, 1999: 435) y la distancia de este respecto a la cláusula de relativo son dos de los factores seleccionados como significativos para la presencia del artículo en las proposiciones oblicuas introducidas por la preposición con.

En lo que se refiere al paralelismo estructural (priming effect), la no presencia del artículo en el antecedente, como en los ejemplos 14 y 15, se muestra como uno de los contextos más favorables a la variante con artículo, con un índice (P.77; $35.4 \%$ ), que apunta en la misma dirección que la categoría "otros" en el factor "determinación" (vid. supra). Por el contrario, los ejemplos alternativos (16 y 17) con presencia del artículo apenas llegan a un $5 \%$ de ocurrencias con la forma innovadora (ejemplos 18 y 19):

(14) [...] lo haré con fina boluntad, con la que pido a Dios guarde a vuestra merced (Cartas desde la otra orilla).

(15) Y tú recibe mi corazón, con el que pido a Dios te guarde muchos años (Cartas desde América).

(16) $[\ldots]$ y vienen muy contentos del cariño y agasajo con que les han tratado aquellos padres (Luengo, Diario, 1767).

(17) [...] no hacen otra cosa que afilar la espada con que ser degollados y torcer la cuerda con que ser ahorcados (Luengo, Diario, 1767).

(18) [...] que es el espíritu con el que en semejantes faenas se corroboraban (La frontera luso-hispana, diario, 1752).

(19) [...] representándoles lo que nos conviene la unión con la que lograremos gozar de lo que Dios nos dio (El castellano de Bilbao en el siglo XVIII).

Por su parte, en nuestro estudio (Blas Arroyo y Vellón, 2017), la presencia del artículo mostraba una clara tendencia: no existe el paralelismo estructural, es decir, el doblado del artículo del antecedente ante el relativo, sino todo lo contrario, la ausencia del artículo favorecía su aparición ante el relativo.

En este caso, el artículo cumple con su función discursiva de recuperar la información conocida, transformando así en temático el referente insertado previamente en el texto y que el hablante interpreta fácilmente como tal, dada la adyacencia del antecedente y la cláusula de relativo. En el otro extremo, la presencia del artículo en el SN antecedente, con su valor como "marca analítica y general de unidad nombradora y potencialmente temática" (Jiménez Juliá, 2007: 132), se muestra como uno de los contextos más firmes para la futura pervivencia de la forma tradicional, en cuanto que el hablante entiende como una redun- 
dancia insistir en la información temática del pronombre relativo, con su función anafórica muy presente dada la proximidad del antecedente.

La distancia es otro de los factores vinculados con el antecedente que aparecen como significativos, de manera que la no adyacencia del antecedente tiene un alto peso explicativo (P.83) en la difusión del relativo con artículo dentro del sistema. Para la codificación de esta variable independiente se distinguieron tres niveles: "adyacencia" (ejemplos 20 y 21), cuando el núcleo del SN antecedente aparece adherido al relativo; "no adyacencia" tanto en los casos de distancia "intermedia" (ejemplos 22 y 23), cuando el núcleo del antecedente está separado del pronombre por algún tipo de complemento (adjetivos, sintagmas preposicionales), como en los de distancia "máxima" (ejemplos 24 y 25), cuando la distancia entre ambos componentes es mayor, por la inserción de otros constituyentes, subordinadas, etc. No obstante, para el análisis de regresión múltiple estos dos últimos se agruparon en un único factor, opuesto a los casos de adyacencia:

(20) [...] debe justificar los motivos con los que pasó su marido a Buenos Aires (Die private Korrespondenz).

(21) [...] estoy bien seguro de la constante amistad con que Vm. corresponde a la que le profeso (Conde de Cervellón. Epistolario).

(22) Repito mi obediencia con la fina voluntad de siempre, con la que pido [...] guarde su vida muchos años (Correspondencia de emigrantes).

(23) [...] no e hallado cosa digna de aprecio con que hacer memoria de Vmd. (Al recibo de esta).

(24) [...] el trabajo de sacar esta copia fiel de la que se dignó remitirme dicho señor para mi consuelo, con la que podrá V. P. y demás reverendos padres quitar [...] (Cartas desde California).

(25) [...] después de la manera tan extraordinaria y cuasi indecente con que el Papa se ha visto tratado del Emperador [...] (Azara. Epistolario).

La bibliografía sobre el tema ha insistido en la relevancia de la distancia para la presencia de las formas con artículo. Bello (1988 [1847]: § 1076) ya había advertido esta asociación, como también Girón Alconchel (2006: 1530) y otros autores (Cortés Rodríguez, 1986: 19; Porto Dapena, 1997: 24; Santana Marrero, 2004: 71; Guzmán Riverón, 2012: 187; Delbecque, 2011: 262, que lo pone en relación con el carácter abstracto del antecedente).

En la presente investigación, sin embargo, se ha ido más allá al plantear el cruce entre este factor y la presencia del artículo en el antecedente, con unos resultados como los que ofrece la Tabla $2^{8}$ :

\footnotetext{
${ }^{8}$ Estos resultados proceden del mismo análisis de regresión que los anteriores, por lo que no se detallan los datos estadísticos que se presentaban en el pie de la Tabla 1. En esta tabla se ofrecen los resultados de la interacción entre factores. El programa de regresión logística permite este análisis que ofrece, como en el caso de la Tabla 1, tanto los porcentajes de aparición — análisis descriptivo, como cálculos probabilísticos (valores $p$ ), es decir, la selección de los factores significativos.
} 


\begin{tabular}{|l|c|c|c|c|c|c|}
\hline \multirow{2}{*}{$\begin{array}{c}\text { PRESENCIA } \\
\text { DEL ARTículo }\end{array}$} & \multicolumn{3}{|c|}{ ADYACENTE } & \multicolumn{3}{c|}{ NO ADYACENTE } \\
\cline { 2 - 7 } & $\mathrm{n}$ & $\%$ & $\mathrm{P}$. & $\mathrm{n}$ & $\%$ & $\mathrm{P}$. \\
\hline Sí & 618 & 91.2 & .31 & 59 & 8.7 & .68 \\
\hline No & 236 & 83.6 & .68 & 46 & 16.3 & .31 \\
\hline Total & 854 & 89 & & 105 & 11 & \\
\hline
\end{tabular}

TABLA 2.-Distribución de la variante artículo + que tras la interacción entre los factores de distancia y presencia del artículo.

Como puede observarse, el programa determina como significativo este cruce, al igual que observábamos en las oblicuas introducidas por la preposición en (Blas Arroyo y Vellón, 2017): la presencia del artículo en el antecedente alejado, como en los ejemplos 26 y 27 , favorece su reproducción en el relativo (P.68), en lo que parece una operación cognitiva de rescate del antecedente y de su actualizador por parte del hablante. Por el contrario, en el contexto adyacente, la presencia del artículo muestra un efecto disimilatorio, por lo que su ausencia es, como vimos, un factor favorecedor de la variante innovadora.

(26) Tengo el honor de dar á V. S. la enhorabuena del feliz succeso que acaba de acontecer, con el que se restituye el sosiego á la Europa y á la Asia (Carta del Almirante Cornísk al Arzobispo de Manila, 1763).

(27) [...] publicando en ellas las noticias más ocultas y esquisitas tocantes a la agricultura, ramo el más interesante de la monarquía, con las que se han ilustrado sobremanera los labradores (José Antonio de Areche, Respuesta del señor Visitador al oficio de Su Excelencia, 1782).

En definitiva, parece que, al menos en los momentos iniciales de este cambio lingüístico, la presencia del artículo ante el pronombre relativo se interpreta como un mecanismo de recuperación funcional del antecedente cuando este presenta dificultades de identificación por parte del hablante, sobre todo por la lejanía con respecto al relativo En este caso, por tanto, contribuye a delimitar y definir la función anafórica del pronombre relativo y su carácter de información temática.

\subsubsection{Sentido de la relativa}

La caracterización semántica de la cláusula de relativo se ha distribuido, para este caso, en dos grupos: a) las que poseen un valor modal (ejemplos 28, 29 y 30) y b) las que presentan otros sentidos, siendo el instrumental (ejemplos 31,32 y 33), el más frecuente en el corpus (en este apartado de "otros sentidos", las relativas con sentido instrumental constituyen el $86 \%, 382$ de 439 
ocurrencias), aunque acompañado para el análisis de regresión por otros significados más ocasionales como los que aparecen en los ejemplos 34 y $35^{9}$ :

(28) No dexarás de estar ya noticioso de mi infelix estado con el que me hallo (Textos para la historia del castellano).

(29) [...] la doncella que la crió reprime su furia con la que le sacó la espina (Sermones del siglo XVIII).

(30) Después del modo indecentísimo con que nos ha tirado en tierra el capitán [...] (Azara. Epistolario).

(31) A esta misión se le ha hecho la gracia de una lancha bien capaz y del todo apareada, con la que han de salir los indios (Cartas desde California).

(32) Avanza sus próximas operaciones y productos con los que comercia (Cartas desde la otra orilla).

(33) Los marcos de plata son 88 de a media libra, que son 44 libras con que se pueden fabricar unos blandones [...] (Cartas desde la otra orilla).

(34) [...] hizo el mal de posición a los pies con una especie de gota con el que me ha tenido sin poderme mover (Azara. Epistolario).

(35) En la orden de S. M. con que V. E. me remite este expediente (Campomanes. Epistolario).

Se trata de un factor que ya se había caracterizado como potencialmente explicativo de la variación en estudios previos (Porto Dapena, 1997: 24-25; Santa Marrero, 2004: 83-84; Balbachán, 2011: 52; y Blas Arroyo y Vellón, 2017 y 2018 se fijan además en el rasgo + humano del antecedente), aunque, como es lógico, con diferentes valores semánticos en función del tipo de preposición. Así, en nuestros trabajo sobre las oblicuas introducidas por la preposición en en el siglo XVIII (2017 y 2018), observamos que el sentido locativo era seleccionado como uno de los contextos más significativos en la difusión del artículo junto al relativo de entre todos los factores considerados en los citados estudios.

A la vista de los datos que figuran en la Tabla 1, resulta evidente que los valores modales favorecen la presencia de la forma tradicional, de tal manera que solo un $5 \%$ de las que poseen este significado están introducidas por la

\footnotetext{
${ }^{9}$ Aunque en el corpus también han aparecido ocurrencias de sentido personal ("da cuenta del revés sufrido por el comerciante con el que estaba en Cádiz", Cartas desde la otra orilla) - 10 muestras-, no se han contemplado en el análisis puesto que parece lógico determinar que la variación, en este caso, sería con cláusulas de relativo introducidas por el pronombre quien. No obstante, en el corpus hay dos ocurrencias de subordinadas relativas de carácter personal introducidas por la forma sin artículo con que: "no tengo persona de satisfacción con que poder pasar a verte" (El hilo que nos une). A pesar de estos ejemplos puntuales, en este caso hay que tener presente dos principios variacionistas (vid. Tagliamonte, 2012): a) marco máximo de la variación: hay que delimitar bien en qué contexto funcional tiene lugar la variación, sin que se mezclen formas que no pueden alternar; y b) responsabilidad ante los datos: hay que analizar la variante que interesa, pero también aquellas que podrían estar en su lugar. En "Vino Miguel, con quien me tomé un café" la variación no es con la forma con que, sino con con el que, lo que no corresponde con el objetivo de este trabajo.
} 
construcción con artículo (P.25); por el contrario, esta última muestra una clara preferencia por el sentido instrumental. En un siglo en el que el cambio presenta todavía un estado incipiente, la forma innovadora solo alcanza el $25 \%$ de todas las relativas con sentido instrumental, pero ello supone una diferencia muy notable con respecto a los contextos modales, lo que se refleja en unos valores probabilísticos (.75 vs. .25) claramente divergentes.

Esta tendencia se vincula con otro factor que muestra cierto grado de coalescencia con él - y de ahí que no se haya considerado en el análisis de regresión-, como es el del carácter referencial del antecedente. La variante innovadora opta por antecedentes concretos (el $24.7 \%$ ), como se observa en el ejemplo 36, frente a la etimológica que prefiere los abstractos (solo el $10 \%$ de tales antecedentes son con artículo - ejemplo 37-). La relación de este factor con el del sentido de la cláusula relativa es diáfana, pues los instrumentales son en su mayoría concretos; por otra parte, los modales suelen ser abstractos (de las 507 ocurrencias con valor modal, solo 5 tienen un antecedente concreto):

(36) [...] que vendido te entregará su líquido producto, con el que procurarás aviarte de todo lo necesario (Cartas desde América).

(37) [...] para que se cumpla con su santísima voluntad, con la que, me puedes creer, me hallo mui conforme (Al recibo de esta).

El hecho de que tanto en el caso de las oblicuas introducidas por en (vid. supra), como en el de las que nos ocupan, el contexto que menos favorece la solución innovadora sea el modal, resulta significativo, máxime cuando, en el otro extremos, los factores en los que se observa la tendencia a las formas con artículo son, respectivamente, el sentido locativo y el instrumental.

En ambos casos hay dos circunstancias que orientan sobre la dirección del cambio que se está operando. Por una parte, se da la coincidencia de que los dos sentidos nombrados se relacionan con antecedentes cuyo núcleo es un nombre de naturaleza semántica preferiblemente concreta, lo que apunta a que el hablante opta por soluciones novedosas en contextos que suponen un menor esfuerzo cognitivo, en cuanto que toman como referencia designaciones semánticas próximas a las experiencia sensitiva. En segundo lugar, la evolución del fenómeno confirma esta línea interpretativa, puesto que el estudio sobre la estructura en que en la época actual (Vellón y Moya, 2017) indica que, precisamente, las cláusulas con sentido modal y temporal, por este orden, constituyen una de las distribuciones privilegiadas de esta fórmula.

\subsubsection{El tipo de relativa}

Este factor distingue entre dos tipos de cláusulas relativas en función de su incidencia en el antecedente. Por una parte, consideramos las relativas especifi- 
cativas (ejemplos 38 y 39), de carácter restrictivo e identificativo. Por otra, las relativas explicativas (ejemplos 40 y 41), que aportan una información complementaria de su referente.

(38) [...] en algunos tributos dados por mí ya ai algunos con los que no puedo contar (Al recibo de esta).

(39) Me llena de gratitud la singular memoria con que los Reyes Nuestros Señores me honran (Campomanes. Epistolario).

(40) [...] difieren de ella en lo largo de su pescuezo, con el que apoyándose contra la tierra recobran fácilmente su situación natural (La frontera luso-hispana).

(41) [...] se acordaron del ídolo del Becerro, con que ofendieron a Dios [...] (Sermones).

Según muestran los datos (ver Tabla 1), la forma con artículo aparece favorecida en las oblicuas explicativas con un índice explicativo muy alto (P.88; $43.8 \%$ ), superior incluso al que obtuvimos en nuestro análisis (Blas Arroyo y Vellón, 2017: 13) de tales cláusulas en las oblicuas introducidas por en (P.65; 21.5 $\%$ ), lo que no hace sino confirmar lo expuesto en todos los trabajos sobre el tema (Porto Dapena, 1997: 24-25; Girón Alconchel, 2006: 1526; Balbachán, 2011: 52).

Autores como Escandell y Leonetti (1989) y López García (1994) ya establecían los paralelismos entre el sistema del artículo y los tipos de relativas. Por ello, hay que recuperar lo comentado en el $\S 5.1 .1$ en torno a la relación entre el antecedente, la presencia del artículo y la distancia con el pronombre relativo:

a) El SN antecedente con artículo y adyacente al pronombre relativo es la distribución menos proclive a la forma innovadora y, en consecuencia, como se ha dicho, la más favorable a las relativas especificativas, puesto que en estas el carácter discriminatorio e identificativo del referente queda garantizado por la proximidad del antecedente, por lo que no se considera tan precisa la presencia del artículo ante el pronombre;

b) por su parte, en el caso de los SN adyacentes, sin artículo, o en los alejados de la cláusula de relativo, con artículo, ambos favorecedores de la forma innovadora, se tiende a las relativas explicativas, de carácter no discriminativo, en las que cobra relevancia el valor identificativo, temático, del antecedente, recuperado a través del artículo, como "marcante de especificidad" (López García, 1994: 432) sobre el que se desarrolla la explicación.

\subsubsection{Otros factores lingüísticos}

El resto de factores lingüísticos comentados anteriormente (vid. supra §4) no ha sido seleccionado como significativo, en algunos casos por la escasa re- 
presentatividad de la muestra (como en el factor de la "polaridad" de la subordinada, pues solo aparecen 3 ocurrencias de polaridad negativa en el corpus); en otros, por la mínima diferencia entre los usos de uno y otro factor, como en la "función del relativo", en la que la frecuencia de la forma con artículo en función de suplemento es de un $15.4 \%$ y la de su presencia como un complemento circunstancial es de un $13.8 \%{ }^{10}$.

En otros factores, sin embargo, algunas diferencias frecuenciales de mayor calado permiten observar diversas tendencias en cuanto a los contextos que favorecerán la expansión de la variante innovadora, que, por otra parte, coinciden con los resultados obtenidos en estudios de temática similar.

Entre ellos, destaca la temporalidad de la cláusula de relativo, con datos como los que se ofrecen en la Tabla $3^{11}$.

\begin{tabular}{|l|c|c|c|c|}
\hline \multirow{2}{*}{ TIEMPO VERBAL } & \multicolumn{2}{|c|}{ RELATIVO SIN ARTícULO } & \multicolumn{2}{c|}{ RELATIVO CON ARTí́CULO } \\
\cline { 2 - 5 } & $\mathrm{n}$ & $\%$ & $\mathrm{n}$ & $\%$ \\
\hline Futuro & 19 & 61 & 12 & 39 \\
\hline Formas no personales & 62 & 100 & 0 & 0 \\
\hline Pasado & 339 & 92.6 & 27 & 7.3 \\
\hline Presente & 384 & 80 & 93 & 20 \\
\hline Otras & 21 & 91 & 2 & 9 \\
\hline
\end{tabular}

TABLA 3.-Frecuencia de aparición de los tiempos verbales en las cláusulas relativas oblicuas introducidas por la preposición con.

El pasado y las formas no personales tienen una presencia absoluta en las cláusulas de relativo sin artículo (42 y 43), es decir, en la forma no marcada de la oposición. Se trata, sobre todo del pasado simple, el tiempo prototípico de la narración, el que define el contenido de la mayoría de los textos integrados en el corpus, cartas en las que un emisor relata sus vicisitudes a un receptor:

(42) $[\ldots]$ hasta en cantidad de veinte mill reales de vellón con las condiziones con que últimamente se hizo (El castellano de Bilbao en el siglo XVIII).

\footnotetext{
${ }^{10}$ Pese a ello, se adivina la tendencia de la forma innovadora hacia la función de suplemento, algo que ya apuntábamos en Vellón y Moya (2017), donde observamos que la forma innovadora muestra preferencia por funciones diferentes al CC.

${ }^{11}$ Los datos de la Tabla 3 proceden de los porcentajes obtenidos en la tabla de contingencia tras la codificación de las ocurrencias. Aunque el programa de regresión no ha seleccionado este factor como significativo desde la óptica variacionista en el siglo XVIII, el análisis descriptivo de porcentajes de aparición apunta a posibles vías futuras del cambio gramatical, como lo demuestran algunos de los estudios citados.
} 
(43) Luego acudieron muchos a buscar un libro con que divertirse (Luengo. Diario).

Por su parte, la exposición personal, siempre más volcada a la subjetividad y al ejercicio estilístico, encuentra en el presente (44) y en el futuro (45) sus canales privilegiados de expresión. Es en estos tiempos donde se aprecia una vía de entrada de la nueva forma, con índices de aparición (20 \% y $39 \%$, respectivamente) que distan mucho de los que caracterizan la presencia de con el que en el resto de tiempos:

(44) [...] 25 pesos que entregarás a Balthasara Mayor, con los que considero satisfecha de algunos gastos [...] (Cartas desde la otra orilla).

(45) $[. .$.$] de sacar esta copia fiel de la que se dignó remitirme dicho señor$ para mi consuelo, con la que podrá V.P. y demás reverendos padres quitar todos los recelos [...] (Cartas desde California).

Mención especial merece el futuro, puesto que en nuestro trabajo sobre las oblicuas introducidas por la preposición en (Blas Arroyo y Vellón, 2017), ya aparecía como la vía de acceso más favorable a la variante innovadora, con un porcentaje de aparición del 35,5\%, muy próximo al obtenido en el caso que nos ocupa. Igualmente, en otros trabajos sobre diacronía (Blas Arroyo y Vellón, 2015: 103), en este caso sobre las perífrasis modales, también pudimos comprobar que este tiempo es un contexto favorable a la difusión de las variantes innovadoras.

Otro de los factores que podrían ser relevantes para la expansión de la solución innovadora ante una muestra más amplia es un segundo ejemplo de paralelismo estructural (priming effect 2), en este caso, la presencia de la preposición con en el antecedente, tal como se muestra en los ejemplos 46 y 47:

(46) ...y se le encomienda con verdadero afecto, con el que queda de Vmd. como siempre este su más apasionado... (Die Private Korrrespondenz spanischer).

(47) Yo me allo bueno a Dios gracias, para servir a v.m. con rendida voluntad, con la que pido a Dios Nuestro Señor me guarde a v.m. muchos años (Correspondencia de emigrantes navarros y guipuzcoanos).

Si bien el programa no lo ha seleccionado como significativo al presentarlo como colineal con el factor distancia del antecedente ${ }^{12}$, los datos apuntan en la dirección comentada, puesto que de las 62 ocurrencias en las que el sintagma del antecedente aparece regido por la preposición con, un 43,5\% $(\mathrm{n}=27)$ corresponden a cláusulas encabezadas por la variante con artículo.

\footnotetext{
${ }^{12}$ Por ello, no es un factor independiente, sino que depende de otro, en este caso, de la distancia del antecedente.
} 
La explicación de estos datos hay que encontrarla en la tendencia del hablante a diferenciar, a través del artículo, dos sintagmas formalmente similares y que aportan la misma información. Ello se comprueba en la fórmula protocolaria epistolar, cuyos núcleos del antecedente son los términos voluntad y afecto (vid. ejemplos 48 y 49). En las 12 ocasiones en las que aparecen precedidas de preposición, se utiliza la variante con artículo. Por su parte, en el corpus, cuando aparecen sin la rección preposicional, en todos los casos se utiliza la forma tradicional (ejemplos 50 y 51):

(48) [...] se le encomiendan con verdadero afecto con el que queda de Vmd como siempre este su más apasionado pariente (Die Private Korrespondenz).

(49) [...] para servir a v.m. con rendida voluntad, con la que pido a Dios Nuestro Señor me guarde a v.m. muchos años (Correspondencia de emigrantes navarros).

(50) [...] te renuevo el afecto con que te ama de corazón tu apasionado padre. (Un epistolario virrenal).

(51) Y uno también mis buenos deseos y segura firme voluntad con que quisiera atender a todos juntos. (Correspondencia de emigrantes navarros y guipuzcoanos).

\subsection{El cambio lingüístico en el eje temporal}

El factor temporal ha sido seleccionado como uno de los factores significativos en el análisis de regresión, tal como sucedió en nuestro trabajo sobre las oblicuas introducidas por en + artículo + que (Blas Arroyo y Vellón, 2017), lo que confirma el proceso diacrónico seguido por el fenómeno a lo largo del siglo XVIII.

En el Gráfico 1 aparece el examen de las frecuencias de aparición de la variante con artículo organizadas por décadas.

En el gráfico se puede apreciar cómo la variante innovadora tiene un uso muy limitado durante las primeras décadas del siglo, en consonancia con los datos de la centuria precedente (vid. supra $\S 1$ ). A partir de ahí se inicia una evolución ascendente hasta llegar a unos índices de aparición del $21 \%$ en la última década. Se trata de una progresión similar a la observada en las relativas oblicuas introducidas por la preposición en a lo largo de esta centuria (Blas Arroyo y Vellón, 2017). En este caso, también el análisis de regresión seleccionó como significativo el factor temporal, y los datos permitieron observar unos índices de aparición de la variante innovadora similares a los que muestra el Gráfico 1, con cifras en torno al $20 \%$ en la última década del siglo. Algunos estudios, como el de Girón Alconchel (2006: 1528), revelan un panorama similar: la forma con artículo comienza a extender su uso a partir de la mitad del 


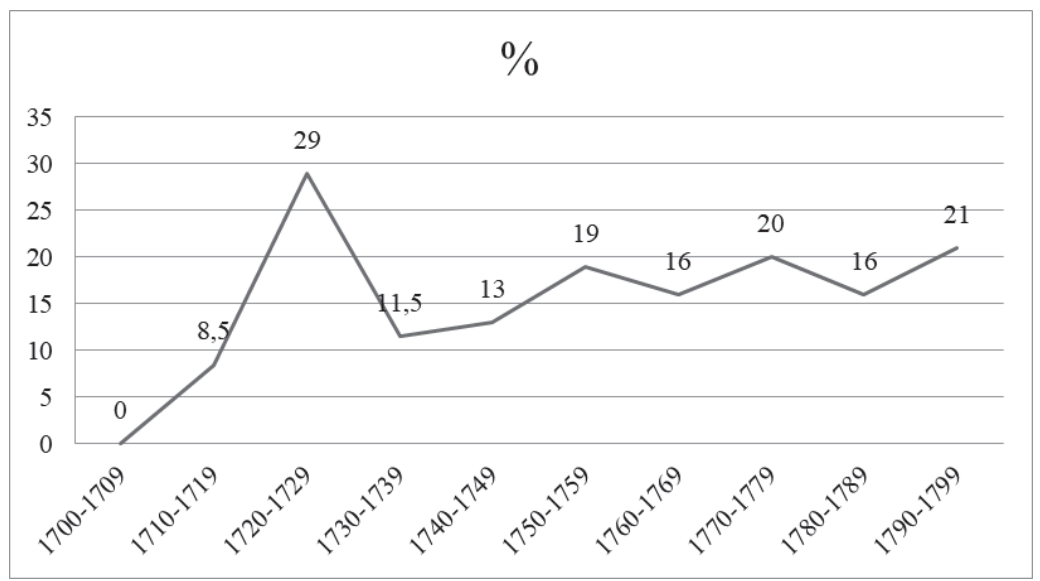

GRÁFICO 1.-Evolución de los índices de frecuencia de la forma con + artículo + que a lo largo del siglo XVIII (\%).

siglo XVIII, con cifras en torno al 15-20\%, que aumentan hacia el final de la centuria (con un $21 \%$ ).

Se observa, por tanto, sobre todo a partir de 1750, la característica "curva en S" (Conde Silvestre, 2005: 153) de los cambios lingüísticos incipientes que se difunden durante un tiempo de manera pausada y continuada en la sociedad y que despliegan correlaciones sociolectales significativas (Nevalainen y RaumolinBrunberg, 1996: 55). A diferencia de otros fenómenos de cambio que se desarrollan en un periodo relativamente corto de tiempo (Martínez, 2001), en este caso la variante innovadora se asienta a lo largo del siglo XVIII con porcentajes de uso cercanos al $20 \%$, que se irán extendiendo en los siglos posteriores, lo que queda para una investigación futura

\section{CONCLUSIONES}

El estudio ha demostrado que en el ámbito de las cláusulas de relativo oblicuas, en concreto en las introducidas por la preposición con a lo largo del siglo XVIII, se está operando el inicio de un cambio lingüístico basado en la aparición del artículo ante el pronombre relativo. Si bien la construcción etimológica (con que) continúa siendo la forma no marcada de la oposición (con un índice frecuencial de uso del $86 \%$ ), en el trabajo se han analizado los contextos a través de los cuales se difunde la variante innovadora, así como las tendencias que se proyectan para investigaciones futuras. 
Entre los factores lingüísticos seleccionados como significativos, destaca en primer lugar la presencia del artículo en el antecedente, puesto que, como señala la mayoría de los estudios sobre el tema, su ausencia es una vía de entrada para las formas con artículo. Sin embargo, en la presente investigación se ha demostrado que, si bien es cierto este contexto que actúa de manera independiente, además interactúa con el de la distancia del referente, de manera que la no presencia del artículo resulta un factor explicativo sobre todo cuando existe adyacencia entre antecedente y relativo. En este caso, el hablante tiende a actualizar el pronombre al observar la indeterminación de su referente. Por otra parte, la presencia del artículo en antecedentes distantes también contribuye a que el hablante reproduzca el artículo ante el relativo, al considerar necesaria la función determinadora dado el alejamiento del referente.

En cuanto al sentido de la cláusula, del mismo modo que en otro lugar comprobamos el significado locativo como el privilegiado como vía de entrada del artículo en las oblicuas con en, el sentido instrumental es el que favorece esta vez la difusión de la variante con el que, frente al sentido modal, más vinculado a la variante tradicional.

El tipo de relativa es otro factor lingüístico seleccionado por el programa. Las cláusulas explicativas suponen uno de los contextos donde mejor se observa la expansión de la variante innovadora, tal como recogen todos los estudios sobre el tema. El motivo hay que buscarlo en la función del "artículo expletivo" y su aportación a un relativo, que, "con una posición de identificación deficiente" (López García, 1994: 442) frente a otros relativos (quien, donde). En el caso de las especificativas, con un anclaje más sólido entre el antecedente y la cláusula de relativo, el aporte identificativo no es tan necesario como en el caso de la explicativas, en las que el anclaje del pronombre es más débil, por lo que el artículo es más necesario, como lo demuestra el caso de el cual, "formante obligatoriamente explicativo" (López García, 1994: 441)

Finalmente, el eje temporal ha demostrado que a partir de 1750 se afianza la presencia de la variante innovadora de un modo gradual pero sólido, con índices de uso que, a finales de la centuria, superan ya el $20 \%$. Queda la tarea de continuar la investigación en los siglos siguientes para evaluar con mayor precisión la proyección de los factores explicativos, la continuidad de las tendencias hasta convertirse o no en significativas, así como la aparición de otros contextos que se suman a los ya existentes. Únicamente a partir de esta tarea se podrá dar cuenta de cuáles han sido las motivaciones de este fenómeno de variación y cambio lingüístico en la historia del español. 


\section{BIBLIOGRAFÍA}

Alarcos Llorach, Emilio (1994): Gramática de la lengua española, Madrid, Espasa-Calpe.

Balbachán, Fernando (2011): "Asimetrías en el uso del artículo definido con cláusulas relativas prepositivas u oblicuas: un análisis semántico-pragmático", Boletín de Lingüística, XXIII, 3536, pp. 31-56.

Bello, Andrés (1988 [1847]): Gramática de la lengua castellana destinada al uso de los americanos, Madrid, Arco/Libros.

Bergs, Alexander (2012): "The uniformitarian principle and the risk of anachronisms in language and social history", en Juan Manuel Hernández Campoy y Juan Conde-Silvestre (eds.), The Handbook of Historical Sociolinguistics, Malden, Wiley-Blackwell, pp. 80-98.

Blas Arroyo, José Luis, Margarita Porcar Miralles y Javier Vellón Lahoz (2013): "Un hito clave en la evolución de las perífrasis modales de infinitivo: análisis sociolingüístico de la alternancia haber deltener que + infinitivo en textos de inmediatez comunicativa del siglo XIX", Revista de Historia de la Lengua Española, 8, pp. 29-62.

Blas Arroyo, José Luis y Javier Vellón Lahoz (2015): "The refuge of a dying variant within the grammar: Patterns of change and continuity in the Spanish verbal periphrasis haber de + infinitive over the past two centuries", Language Variation and Change, 27, pp. 89-116.

Blas Arroyo, José Luis y Javier Vellón Lahoz (2017): "En los albores de un cambio lingüístico: factores condicionantes y fases en la inserción del artículo en las relativas oblicuas del siglo XVIII", Zeitschrift für romanische Philologie, 133 (2), pp. 492-529.

Blas Arroyo, José Luis y Javier Vellón Lahoz (2018): "On the trail of grammaticalisation in progress: is el que a compound relative pronoun in Spanish prepositional relative clauses?", Probus, 30 (1), pp. 1-45, <https://doi.org/10.1515/probus-2017-0010>.

Bosque, Ignacio y Juan Carlos Moreno (1990): "Las construcciones con lo y la denotación del neutro", Lingüística, 2, pp. 5-50.

Briz, Antonio y Manuel Prunyonosa (1987): Sintaxi i semántica de l'article, València, Universitat de València.

Brucart, José María (1999): "La estructura del sintagma nominal: las oraciones de relativo", en Ignacio Bosque y Violeta Demonte (eds.), Gramática descriptiva de la lengua española, Madrid, Espasa-Calpe, pp. 395-522.

Claes, Jeroen (2014): "La pluralización de haber presentacional en el español de La Habana: interacción entre las restricciones cognitivas", Revista Internacional de Lingüística Iberoamericana, 23, pp. 165-187.

Conde Silvestre, Juan Camilo (2005): Sociolingüística histórica, Madrid, Gredos.

Cortés Rodríguez, Luis (1986): "Alternancia de los relativos donde: quelel que: el cual en el español hablado", Revista Española de Lingüística Aplicada (2), pp. 9-22.

Delbecque, Nicole (2011): "Alternancia de los pronombres relativos el que y que tras preposición: especificación vs. apoyo discursivo", en Ana María Cestero, Isabel Molina Martos y Florentino Paredes García, Congreso Internacional de la Asociación de Lingüística y Filología de América Latina. "La lengua, lugar de encuentro", Alcalá de Henares, 6-9 de junio de 2011, Alcalá de Henares, Universidad de Alcalá, pp. 253-263.

Eberenz, Rolf (2000): El español en el otoño de la Edad Media. Sobre el artículo y los pronombres, Madrid, Gredos.

Elspass, Stephan (2012): "Between linguistic creativity and formulaic restriction: Cross-linguistics perspectives on nineteenth-century lower class writers' private letters", en Marina Dossena y Gabriella del Lungo (eds.), Letter Writing in Late Modern Europe, Pragmatics \& Beyond New Series (218), Amsterdam/Philadelphia, John Benjamins Pub. Co., pp. 45-64.

Elvira, Javier (1985): Lingüística histórica y cambio gramatical, Madrid, Síntesis.

Escandell, M. ${ }^{a}$ Victoria y Manuel Leonetti (1989): "Notas sobre la aposición nominal", Revista de Filología Española, LXIX, pp. 163-172. 
García García, Serafina (1990): Los transcriptores oracionales en la obra histórica alfonsí. Estudio de sintaxis funcional, Oviedo, Universidad de Oviedo.

Gili Gaya, Samuel (1970): Curso superior de sintaxis española, Barcelona, Vox.

Girón Alconchel, José Luis (2006): "Las oraciones de relativo II. Evolución del relativo compuesto EL QUE, LA QUE, LO QUE", en Concepción Company (dir.), Sintaxis histórica de la lengua española. Segunda parte: la frase nominal, México, UNAM/Fondo de Cultura Económica, vol. 2, pp. 1477-1590.

Guzmán Riverón, Martha (2012): "El artículo en las relativas oblicuas [prep. + (art. definido) + que] en textos americanos del siglo XVIII", Cuadernos dieciochistas, 13, pp. 175-208.

Hernández Alonso, César (1986): Gramática funcional del español, Madrid, Gredos.

Jiménez Juliá, Tomás (2007): Aspectos gramaticales de la frase nominal en español, Anexo 60 de la revista Verba, Santiago de Compostela, Universidad de Santiago de Compostela.

Johnson, Daniel Ezra (2009): "Getting off the GoldVarb Standard: Introducing Rbrul for MixedEffects Variable Rule Analysis", Language and Linguistics Compass, 3 (1), pp. 359-383.

Keniston, Hayward (1937): The syntax of Castilian prose. The Sixteenth century, Chicago, The University of Chicago Press.

Labov, William (1972): Sociolinguistics Patterns, Philadelphia, University of Penssylvania Press.

Labov, William (1996): Principios del cambio lingüístico, Madrid, Gredos.

Lapesa, Rafael (2000): " $E l$, la, lo como antecedente de relativo en español", en Rafael Cano y María Teresa Echenique (eds.), Estudios de morfosintaxis histórica del español, Madrid, Gredos, pp. 388-401.

Leonetti, Manuel (1999): "El artículo", en Ignacio Bosque y Violeta Demonte (eds.), Gramática descriptiva de la lengua española, Madrid, Espasa-Calpe, pp. 787-890.

Lope Blanch, Juan Miguel (1998): "Los relativos en la segunda carta de Hernán Cortés", en García, Claudia et alii (coords.), Actas del IV Congreso Internacional de Historia de la Lengua Española, La Rioja, 1-5 de abril de 1997, Logroño, Universidad de la Rioja, vol. I, pp. $573-580$

López García, Ángel (1994): Gramática del español. I La oración compuesta, Madrid, Arco/Libros.

Marcos Marín, Eugenio (1980): Curso de gramática española, Madrid, Cincel.

Martínez, Glenn (2001): "Política lingüística y contacto social en el español México-tejano: la oposición -ra y -se en Tejas", Hispania, 84 (1), pp. 114-124.

Martínez, José Antonio (1989): El pronombre II: numerales, indefinidos y relativos, Madrid, Arco/Libros.

Nevalainen, Terttu y Helena Raumolin-Brunberg (1996): Sociolinguistics and Language History. Studies Based on the Corpus of Early English Correspondence, Amsterdam, Atlanta.

Ojea, Ana I. (1992): Los sintagmas relativos en inglés y en español, Oviedo, Universidad de Oviedo.

Oesterreicher, Wulf (2004): "Textos entre inmediatez y distancia comunicativas. El problema de lo hablado escrito en el Siglo de Oro", en Rafael Cano (coord.), Historia de la lengua espanola, Barcelona, Ariel, pp. 729-769.

Poplack, Shana y Nathalie Dion (2009): "Prescription vs. praxis: The evolution of future temporal reference in French", Language 85 (3), pp. 557-587.

Poplack, Shana y Elisabete Malvar (2007): "Elucidating the transition period in linguistic change: The expression of the future in Brazilian Portuguese", Probus, 19, pp. 121-169.

Porto Dapena, José Álvaro (1997): Relativos e interrogativos, Madrid, Arco/Libros.

Rivero, María Luisa (1991): Las construcciones de relativo, Madrid, Taurus.

Sánchez Lancis, Carlos (2012): "Periodización y cambio gramatical: el siglo XVIII, ¿frontera temporal del español?", en M. ${ }^{a}$ Teresa García-Godoy (ed.), El español del siglo XVIII. Cambios 
diacrónicos en el primer español moderno, Fondo hispánico de lingüística y filología, vol. 10, Bern, Peter Lang, pp. 21-51.

Santana Marrero, Juana (2004): "Preposición + (artículo) + que relativo: análisis en la norma lingüística culta panhispánica”, Boletín de Lingüística, 21, pp. 66-91.

Tagliamonte, Sali (2012): Variationist Sociolinguistics. Change, Observation, Interpretation, Oxford, Wiley-Blackwell.

Trujillo, Ramón (1990): "Sobre la supuesta despronominalización del relativo", Estudios de lingüística de la Universidad de Alicante, 6, pp. 23-46.

Vellón Lahoz, Javier y Rosana Moya Isach (2017): "Pervivencia de las relativas oblicuas sin artículo: factores y contextos condicionantes”, Spanish in Context, 14 (3), pp. 464-486.

Fecha de recepción: 8 de junio de 2017

Fecha de aceptación: 18 de septiembre de 2017 